\title{
A systematic evaluation of intraoperative white matter tract shift in pediatric epilepsy surgery using high-field MRI and probabilistic high angular resolution diffusion imaging tractography
}

\author{
*Joseph Yuan-Mou Yang, PhD, ${ }^{1-4}$ Richard Beare, PhD, ${ }^{2,7}$ Marc L. Seal, PhD, ${ }^{2,4}$ \\ A. Simon Harvey, MD, ${ }^{3-5}$ Vicki A. Anderson, PhD, ${ }^{4,6,8,9}$ and Wirginia J. Maixner, MBBS ${ }^{1,3}$ \\ Departments of ${ }^{1}$ Neurosurgery, ${ }^{5}$ Neurology, and ${ }^{8}$ Psychology, Royal Children's Hospital; ${ }^{2}$ Developmental Imaging Group and \\ ${ }^{3}$ Neuroscience Research Group, ${ }^{6}$ Clinical Sciences Theme, Murdoch Childrens Research Institute; ${ }^{4}$ Department of Paediatrics \\ and ${ }^{9}$ School of Psychological Sciences, University of Melbourne; and ${ }^{7}$ Department of Medicine, Monash University, Melbourne, \\ Victoria, Australia
}

\begin{abstract}
OBJECTIVE Characterization of intraoperative white matter tract (WMT) shift has the potential to compensate for neuronavigation inaccuracies using preoperative brain imaging. This study aimed to quantify and characterize intraoperative WMT shift from the global hemispheric to the regional tract-based scale and to investigate the impact of intraoperative factors (IOFs).
\end{abstract}

METHODS High angular resolution diffusion imaging (HARDI) diffusion-weighted data were acquired over 5 consecutive perioperative time points $\left(M R_{1}\right.$ to $M R_{5}$ ) in 16 epilepsy patients (8 male; mean age 9.8 years, range 3.8-15.8 years) using diagnostic and intraoperative 3-T MRI scanners. $\mathrm{MR}_{1}$ was the preoperative planning scan. $\mathrm{MR}_{2}$ was the first intraoperative scan acquired with the patient's head fixed in the surgical position. $\mathrm{MR}_{3}$ was the second intraoperative scan acquired following craniotomy and durotomy, prior to lesion resection. $\mathrm{MR}_{4}$ was the last intraoperative scan acquired following lesion resection, prior to wound closure. $\mathrm{MR}_{5}$ was a postoperative scan acquired at the 3-month follow-up visit. Ten association WMT/WMT segments and 1 projection WMT were generated via a probabilistic tractography algorithm from each MRI scan. Image registration was performed through pairwise MRI alignments using the skull segmentation. The $M R_{1}$ and $M R_{2}$ pairing represented the first surgical stage. The $M R_{2}$ and $M R_{3}$ pairing represented the second surgical stage. The $\mathrm{MR}_{3}$ and $\mathrm{MR}_{4}$ (or $\mathrm{MR}_{5}$ ) pairing represented the third surgical stage. The WMT shift was quantified by measuring displacements between a pair of WMT centerlines. Linear mixed-effects regression analyses were carried out for 6 IOFs: head rotation, craniotomy size, durotomy size, resected lesion volume, presence of brain edema, and CSF loss via ventricular penetration.

RESULTS The average WMT shift in the operative hemisphere was $2.37 \mathrm{~mm}$ (range 1.92-3.03 $\mathrm{mm}$ ) during the first surgical stage, $2.19 \mathrm{~mm}$ (range 1.90-3.65 mm) during the second surgical stage, and $2.92 \mathrm{~mm}$ (range 2.19-4.32 mm) during the third surgical stage. Greater WMT shift occurred in the operative than the nonoperative hemisphere, in the WMTs adjacent to the surgical lesion rather than those remote to it, and in the superficial rather than the deep segment of the pyramidal tract. Durotomy size and resection size were significant, independent IOFs affecting WMT shift. The presence of brain edema was a marginally significant IOF. Craniotomy size, degree of head rotation, and ventricular penetration were not significant IOFs affecting WMT shift.

CONCLUSIONS WMT shift occurs noticeably in tracts adjacent to the surgical lesions, and those motor tracts superfi-

ABBREVIATIONS $A B=$ anterior bundle; $\mathrm{AH}-\mathrm{CING}=$ anterior horizontal $\mathrm{CING} ; \mathrm{CING}=$ cingulum; $\mathrm{CSD}=$ constrained spherical deconvolution; $\mathrm{DNET}=$ dysembryoplastic neuroepithelial tumor; $\mathrm{DTI}=$ diffusion tensor imaging; $\mathrm{EPI}=$ echo-planar imaging; $\mathrm{ET}-\mathrm{CO}_{2}$ end-tidal carbon dioxide; $\mathrm{FOD}=$ fiber orientation distribution; $\mathrm{Fx}=$ fornix; $\mathrm{HARDI}=$ high angular resolution diffusion imaging; ICV = intracranial volume; IFOF = inferior fronto-occipital fasciculus; ILF = inferior longitudinal fasciculus; IOF = intraoperative factor; $\mathrm{MB}=$ middle bundle; $\mathrm{OR}=$ optic radiation; $\mathrm{PB}=$ posterior bundle; $\mathrm{PH}-\mathrm{CING}=$ parahippocampal CING; $\mathrm{PS}=$ probability of superiority; $\mathrm{PT}=$ pyramidal tract; $\mathrm{ROI}=$ region of interest; $R_{\text {mar }}^{2}=$ marginal $R^{2} ; S E$ = standard error; $S L F=$ superior longitudinal fasciculus; $U F=$ uncinate fasciculus; WMT = white matter tract. SUBMITTED May 28, 2016. ACCEPTED November 15, 2016.

INCLUDE WHEN CITING Published online March 17, 2017; DOI: 10.3171/2016.11.PEDS16312.

* Drs. Yang and Beare contributed equally to this work. 


\begin{abstract}
cially placed in the operative hemisphere. Intraoperative probabilistic HARDI tractography following craniotomy, durotomy, and lesion resection may compensate for intraoperative WMT shift and improve neuronavigation accuracy.

https://thejns.org/doi/abs/10.3171/2016.11.PEDS16312
\end{abstract}

KEY WORDS diffusion tractography; high angular resolution diffusion imaging; intraoperative high-field MRI; pediatric epilepsy surgery; probabilistic tractography algorithm; white matter tract shift

$\mathrm{T}$ HE major shortcoming of surgical neuronavigation systems utilizing preoperatively acquired images is the inability to account for intraoperative brain shift. ${ }^{33,37,42,47,49}$ Brain shift occurs as a result of various interactive factors, such as gravity, brain edema, loss of CSF, and lesion resection. ${ }^{33,37,42,47}$ While most notable at the cortical surface, it can also occur at the white matter level. Understanding brain shift has important practical implications, including maximizing lesion resection and preserving eloquent cortical regions and white matter tracts (WMTs). ${ }^{42,49}$

Diffusion tractography is an MRI postprocessing technique that provides virtual reconstructions of WMT anatomy based on brain diffusion information.,36 The technique adopted in neurosurgery typically involves diffusion tensor imaging (DTI) data acquisition and a deterministic tractography algorithm,,$^{10,12,38}$ but this approach is unable to accurately model diffusion over crossing fiber regions..$^{10,29,38,55}$ More sophisticated diffusion-weighted MRI sequences ${ }^{1,3,21,53,54,56,57}$ and tractography algorithms $\mathrm{s}^{6,7,44,52,55}$ have been developed to optimize resolution and estimation of fiber orientation over these regions.

High angular resolution diffusion imaging (HARDI) data are acquired using a higher diffusion gradient (bvalue $\geq 3000 \mathrm{sec} / \mathrm{mm}^{2}$ ) and with a greater number of noncollinear diffusion directions ( $\geq 45$ directions) than DTI data. ${ }^{1,32,54,55}$ HARDI acquisition therefore captures more high angular frequency details of the diffusion-weighted signal than a DTI acquisition, enabling a better estimate of the fiber orientation distribution (FOD) in each MRI voxel. ${ }^{53,54}$ Further, the use of a probabilistic tractography algorithm addresses the limitation of characterizing regions with uncertain diffusion estimated by providing the tractography results in the form of a probability distribution rather than a single best-fit estimate. ${ }^{6,744,55}$ Combined HARDI data acquisition and a probabilistic tractography algorithm (abbreviated as "probabilistic HARDI tractography" for the rest of this paper) is widely accepted as a more suitable method for in vivo WMT modeling and estimation for anatomical connectivity across the whole brain than DTI. $6,7,25,38,52$

The emergence of operating suites with built-in MRI scanners, or intraoperative MRI, offers a brain shift compensation strategy by providing updated brain anatomy images when necessary. ${ }^{18,30,37}$ Diffusion tractography complements intraoperative MRI by permitting assessments of brain shift occurring at the WMT level. .1,42,43,49 $^{3}$

Existing studies concerning evaluation of intraoperative WMT shift focused on adult brain tumor cases and single-WMT reconstruction, usually the pyramidal tract (PT), using deterministic DTI tractography. ${ }^{31,42,43,49}$ Evaluations were usually confined to analysis of preoperative MRI and an intraoperative postresection MRI. The im- pacts of different intraoperative factors (IOFs) upon the WMT shift were inadequately addressed due to the limited data available. Only 1 study addressed the impact of dural opening on PT shift using additional intraoperative MRI acquisition in a patient subset. ${ }^{49}$

In this study, we evaluated intraoperative WMT shift in the context of pediatric epilepsy surgery. We used data acquired from comparable preoperative, intraoperative, and postoperative high-field (3-T) MRI systems and generated several WMTs using probabilistic HARDI tractography.

This study had 2 aims. 1) The first aim was to quantify WMT shift across 3 surgical stages. Shift was assessed with respect to the operative and nonoperative hemispheres, proximity to the resection site, and the superficial compared with deeper segment of the WMT studied. We anticipated that greater WMT shift would be evident in the later surgery stages, in the operative hemisphere, closer to the resection site, and in the superficial rather than deeper WMT segments. 2) The second aim was to investigate the effects of 6 IOFs on WMT shifts: head rotation, craniotomy size, durotomy size, resected lesion volume, CSF loss via ventricular penetration, and brain edema. We predicted that the magnitude of WMT shift would be positively associated with each IOF, but the direction of the shift would be variable.

\section{Methods \\ Patient Population}

We recruited 16 children undergoing surgery for drugresistant focal epilepsy at the Royal Children's Hospital, Melbourne, Australia ( 8 male; mean age 9.8 years, range $3.8-15.8$ years) for this prospective study. The study was approved by the hospital's human research and ethics committee. Prior to study commencement, signed, informed consent was obtained from the patients' parents or guardians and from cognitively competent patients older than 12 years old.

\section{MRI Acquisition and Data Processing}

Up to 5 perioperative MRI scans were acquired for each patient (Fig. 1), as follows:

- $\mathrm{MR}_{1}$ - preoperative MRI used for diagnosis and surgical planning, acquired several months before surgery (mean 3.8 months before surgery, range 4 days-6.8 months);

- $\mathrm{MR}_{2}$ - first intraoperative MRI scan, acquired with the patient's head fixed in the surgical position, under anesthesia, prior to craniotomy;

- $\mathrm{MR}_{3}$ - second intraoperative MRI scan, acquired following craniotomy and durotomy, prior to lesion resection; 

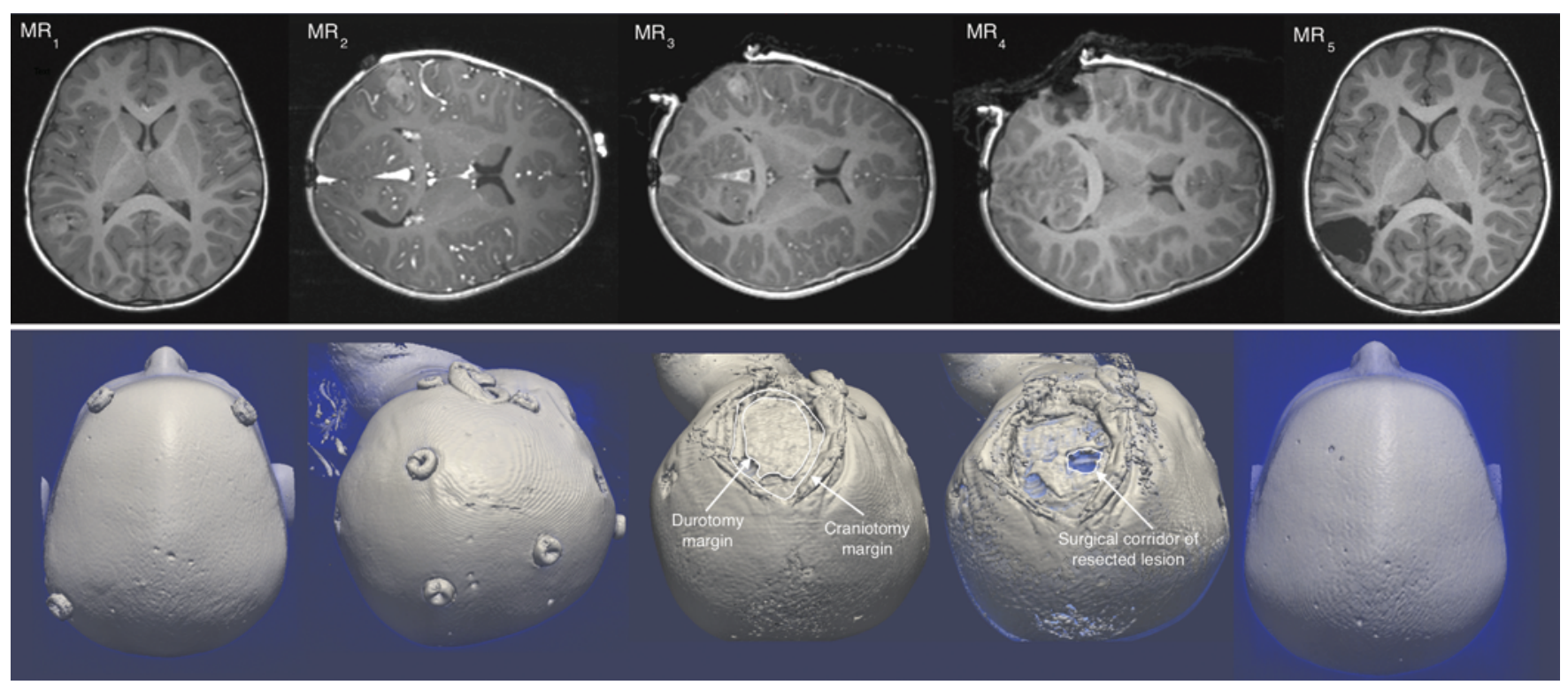

FIG. 1. Case 4. Axial T1-weighted MR images (upper) and corresponding 3D rendered skull images (lower) across 5 consecutive perioperative time points. The surgery was performed for resection of an angular gyrus DNET. The MR images are shown in anatomical convention to match the $3 \mathrm{D}$ skull renderings. $M R_{1}$ is the preoperative scan (head in a supine neutral position); $M R_{2}$, the intraoperative, pre-scalp incision scan (head rotated $90^{\circ}$ ); $\mathrm{MR}_{3}$, the intraoperative, post-cranial and dural opening scan; $\mathrm{MR}_{4}$, the intraoperative, post-lesion resection scan; $\mathrm{MR}_{5}$, the 3-month postoperative scan (head in a supine neutral position). The donutshaped markers on the 3D rendered skull images are radiopaque fiducial markers used for surgical navigation registration. The frontal and occipital skull indentations are artifacts arising from pin fixation of the surgical head frame. Figure is available in color online only.

- $\mathrm{MR}_{4}$-third intraoperative MRI scan, acquired following lesion resection, prior to dura and skull closure;

- $\mathrm{MR}_{5}$ - postoperative MRI scan, acquired at the 3-month follow-up visit (mean 3.4 months after surgery, range 2.6-4.4 months).

$\mathrm{MR}_{1}$ and $\mathrm{MR}_{5}$ were acquired using a diagnostic $3 \mathrm{~T}$ MRI scanner (Siemens Magnetom Trio) with a 32-channel head coil. $\mathrm{MR}_{2}, \mathrm{MR}_{3}$, and $\mathrm{MR}_{4}$ were acquired using a 3-T movable Siemens intraoperative MRI scanner (IMRIS, Manitoba, Canada) with an 8-channel head coil in the operating room with radiofrequency shielding.

The MRI sequences required for this study included a T1-weighted anatomical sequence and a HARDI diffusion sequence. The anatomical sequence acquired was a T1weighted high-resolution magnetization prepared gradient echo (MPRAGE) (for $\mathrm{MR}_{1}$ and $\mathrm{MR}_{5}$ scans, $256 \times 256$ acquisition matrix, FOV 250, $0.8 \mathrm{~mm}^{3}$ isotropic voxels, TR $1900 \mathrm{msec}$, TE $2.69 \mathrm{msec}$; for $\mathrm{MR}_{2}, \mathrm{MR}_{3}$, and $\mathrm{MR}_{4}$ scans, $256 \times 256$ acquisition matrix, FOV 256, $1.0 \mathrm{~mm}^{3}$ isotropic voxels, TR $1800 \mathrm{msec}$, TE $2.19 \mathrm{msec}$ ). The HARDI diffusion data were acquired using the pulsed gradient spin echo (PGSE) sequence with a single-shot spin-echo, echoplanar imaging (EPI) read out, 60 noncollinear DW directions $\left(b=3000 \mathrm{sec} / \mathrm{mm}^{2}\right), 7 \mathrm{~b} 0$ reference volumes, 54 contiguous slices, $2.3 \mathrm{~mm}^{3}$ isotropic voxels, TR $7600 \mathrm{msec}$, TE $110 \mathrm{msec}$. Additional T2-weighted turbo-spin echo (TSE) sequence with multiplanar reconstructions and case-specific T2-weighted 3D volumetric sequences were acquired at $\mathrm{MR}_{1}$ and $\mathrm{MR}_{5}$ (as per routine epilepsy workup and follow-up). The T2 volumetric sequences were also acquired at $\mathrm{MR}_{2}$ (to assist surgical planning and intraoperative neuronavigation by fusing with the $\mathrm{T} 1$ volumet- ric data) and at $\mathrm{MR}_{4}$ (to assist postresection evaluation). They were the fluid attenuation inverse recovery (FLAIR) sequence, the dual inverse recovery (DIR) sequence, or the sampling perfection with application optimized with contrasts using different flip angle evolution (SPACE) sequence. The total acquisition times were approximately 45 minutes for $\mathrm{MR}_{1}$ and $\mathrm{MR}_{5}, 35$ minutes for $\mathrm{MR}_{2}, 20$ minutes for $\mathrm{MR}_{3}$, and 35 minutes for $\mathrm{MR}_{4}$. $\mathrm{MR}_{3}$ was the only study-specific intraoperative scanning session. The HARDI acquisitions were study specific, and increased $\mathrm{MR}_{2}$ and $\mathrm{MR}_{4}$ by about 10 minutes each. The added risks of scans to the patients for this study were considered small and included in the hospital's ethics committee-approved Parent/Guardian Information Statement Form.

The fiber orientation distribution (FOD) was estimated at each voxel of the HARDI diffusion data using the constrained spherical deconvolution (CSD) model of the MRtrix software package, version 0.2.12 (Brain Research Institute, Melbourne, Australia; software package download available at http://www.nitrc.org/projects/mrtrix/; user documentation can be found at http://jdtournier.github.io/ mrtrix-0.2/index.html). ${ }^{52-54}$

$\mathrm{MR}_{1}$ through $\mathrm{MR}_{5}$ scans were aligned using an inhouse-developed automated registration procedure based on skull segmentation. Subvoxel, submillimeter accuracy of the registration procedure has been demonstrated in our previous publication. ${ }^{5}$

\section{White Matter Fiber Tracking: Probabilistic HARDI Tractography}

The following WMTs were reconstructed from HARDI 
TABLE 1. Summary of patients' demographic and clinical characteristics and intraoperative information

\begin{tabular}{|c|c|c|c|c|c|c|c|c|c|c|c|c|}
\hline $\begin{array}{c}\text { Case } \\
\text { No. }\end{array}$ & $\begin{array}{c}\mathrm{Pt} \\
\text { Age } \\
\text { (yrs) }\end{array}$ & Pathology & $\begin{array}{c}\text { Head } \\
\text { Position } \\
\left(^{\circ}\right)\end{array}$ & $\begin{array}{l}\text { Lesion } \\
\text { Side } \\
\text { (Lt/Rt) }\end{array}$ & $\begin{array}{c}\text { Lesion } \\
\text { Site }\end{array}$ & $\begin{array}{c}\text { Resect } \\
\text { Lesion } \\
\text { Vol }\left(\mathrm{cm}^{3}\right)\end{array}$ & $\begin{array}{c}\text { Cx } \\
\text { Size } \\
\left(\mathrm{cm}^{2}\right)\end{array}$ & $\begin{array}{l}\text { Dx } \\
\text { Size } \\
\left(\mathrm{cm}^{2}\right)\end{array}$ & $\begin{array}{c}\text { Avg. } \\
\mathrm{ET}^{-\mathrm{CO}_{2}} \\
(\mathrm{~mm} \mathrm{Hg})\end{array}$ & $\begin{array}{c}\text { Total IV } \\
\text { Steroid } \\
\text { Used (mg) }\end{array}$ & $\begin{array}{c}\text { Ventricle } \\
\text { Entered } \\
(\mathrm{Y} / \mathrm{N})\end{array}$ & $\begin{array}{c}\text { Brain } \\
\text { Edema } \\
(\mathrm{Y} / \mathrm{N})\end{array}$ \\
\hline 1 & 13.42 & Gliosis & 60 & $\mathrm{Lt}$ & $\mathrm{F}$ & 3.9 & 81.0 & 15.4 & 36.5 & 12 & $\mathrm{~N}$ & $\mathrm{~N}$ \\
\hline 2 & 13.42 & FCD & 90 & $\mathrm{Lt}$ & $\mathrm{T}$ & 16.5 & 55.3 & 48.0 & 37.3 & 16 & $\mathrm{~N}$ & $\mathrm{~N}$ \\
\hline 3 & 3.75 & TSC & 5 & Rt & $F, T^{*}$ & $9.5^{*}$ & 48.8 & $5.0+\S$ & 38.7 & 10.6 & $\mathrm{~N}$ & $Y$ \\
\hline 4 & 15.83 & DNET & 90 & Rt & $\mathrm{F}$ & 13.5 & 32.4 & 27.5 & 34.3 & 11 & $\mathrm{~N}$ & $\mathrm{~N}$ \\
\hline 5 & 15.50 & Chronic encephalitis & 90 & $\mathrm{Lt}$ & $\mathrm{F}$ & 12.4 & 82.5 & 73.5 & 36.7 & 16 & $\mathrm{~N}$ & $\mathrm{~N}$ \\
\hline 6 & 12.25 & Gliosis & 90 & Rt & $\mathrm{T}$ & 20.7 & 33.8 & 28.0 & 34.7 & 10 & Y & $\mathrm{N}$ \\
\hline 7 & 4.83 & FCD w/ DVA & 90 & $\mathrm{Lt}$ & $\mathrm{F}$ & 3.9 & 37.5 & 31.5 & 39.7 & 8 & $\mathrm{~N}$ & $\mathrm{~N}$ \\
\hline 8 & 10.17 & Nonspecific & 90 & Rt & $\mathrm{T}$ & 46.7 & 30.0 & 24.8 & 38.5 & 16 & Y & $\mathrm{N}$ \\
\hline 9 & 3.92 & DNET & 90 & $\mathrm{Lt}$ & TPOJ & 7.3 & 29.3 & 22.5 & 41.0 & 6 & $\mathrm{~N}$ & $\mathrm{~N}$ \\
\hline 10 & 4.17 & TSC & 90 & $\mathrm{Rt}$ & $F$ & 7.8 & 36.0 & $5.0 \S$ & 34.3 & 8 & $\mathrm{~N}$ & $Y$ \\
\hline 11 & 3.83 & FCD & 0 & $\mathrm{Lt}$ & $F$ & 76.4 & 65.0 & $5.0 \S$ & 42.0 & 3 & $\mathrm{~N}$ & $Y$ \\
\hline 12 & 4.50 & FCD & 90 & $\mathrm{Lt}$ & $\mathrm{F}, \mathrm{T}, \mathrm{P}, \mathrm{I}^{*}$ & $163.3^{*}$ & 74.8 & $5.0 \S$ & 35.5 & 6 & $\mathrm{~N}$ & Y \\
\hline 13 & 10.92 & DNET & 120 & Rt & 0 & 7.0 & 22.0 & $5.0 \S$ & 36.3 & 4 & $\mathrm{~N}$ & $Y$ \\
\hline 14 & 13.25 & DNET & 90 & $\mathrm{Lt}$ & TPOJ & 5.2 & 19.6 & $3.1 \S$ & 32.3 & 8 & $\mathrm{~N}$ & $Y$ \\
\hline 15 & 15.42 & TSC & 120 & Rt & 0 & 4.6 & 20.3 & $5.0 \S$ & 34.3 & 16 & $\mathrm{~N}$ & Y \\
\hline 16 & 12.17 & DNET & 120 & $\mathrm{Rt}$ & TPOJ & 24.2 & 35.8 & 27.0 & 41.7 & 8 & $\mathrm{~N}$ & $\mathrm{~N}$ \\
\hline
\end{tabular}

$\mathrm{Cx}=$ craniotomy; DVA = developmental venous anomaly; $\mathrm{Dx}=$ durotomy; $E C=$ significant extra-axial collections/air; $F=$ frontal; FCD = focal cortical dysplasia; $\mathrm{I}=$ insula; $\mathrm{ICH}=$ intracerebral hematoma; $\mathrm{IV}=$ intravenous; $\mathrm{N}=$ no; $\mathrm{O}=$ occipital; $\mathrm{P}=$ parietal; $\mathrm{pt}$ = patient; resect = resected; $\mathrm{T}=$ temporal; $\mathrm{TSC}=$ tuberous sclerosis complex; TPOJ = temporoparietooccipital junction; $Y=$ yes.

* Multiple cortical lesions were resected in these cases. Combined volumes of these lesions are represented here.

$\dagger$ This case involved a separate craniotomy performed in the left hemicranium, without dural opening and brain resection.

$\ddagger$ Temporoparietooccipital junction location included lesions involving either the subcortical white matter or cortical regions of the supramarginal gyrus, angular gyrus, posterior portions of the middle and inferior temporal gyri, or the fusiform gyrus.

$\S$ Slit durotomy was performed in these cases.

diffusion data of each scan: pyramidal tract (PT), superior longitudinal fasciculus (SLF), optic radiation (OR), inferior longitudinal fasciculus (ILF), inferior fronto-occipital fasciculus (IFOF), uncinate fasciculus (UF), cingulum (CING), and fornix (Fx). The OR was generated in 3 bundles: anterior, middle, and posterior (AB-, MB-, and PB-OR). The CING was generated as an anterior horizontal (AH-CING) and a parahippocampal (PH-CING) segment.

All tracts were reconstructed using a knowledge-based, multiple region-of-interest (ROI) approach. The ROIs were defined manually by an experienced tractographer (the first author, J.Y.M.Y.). All tractography streamlines originated from the seed ROI and passed through a series of inclusion ROIs. Exclusion ROIs were placed in regions through which the studied WMT was known not to pass. Supplementary Table 1 summarized the ROIs defined for each WMT and WMT segments. Tractography was performed using the probabilistic tractography algorithm implemented in the MRtrix software package with default parameters (radius curvature $1 \mathrm{~mm}$; stepsize 0.2 $\mathrm{mm}$; minimum and maximum lengths 10 and $200 \mathrm{~mm}$, respectively; FOD amplitude threshold 0.1 defined tracking termination). The reliability of this technical approach had previously been demonstrated. ${ }^{22,23,52}$

\section{Intraoperative Factor Definitions}

The 6 intraoperative factors (IOFs) studied were:
- Head rotation angle (degrees): estimated angle of head rotation from the vertical line.

- Craniotomy size: measured from the patient's bone flap intraoperatively and expressed as an estimated surface area in square centimeters $\left(\mathrm{cm}^{2}\right)$.

- Durotomy size: measured from the patient's dural opening intraoperatively and expressed as an estimated surface area in square centimeters $\left(\mathrm{cm}^{2}\right)$. If only slit dural opening was performed due to presence of brain edema, the length of the opening was measured and multiplied by an assumed 1-cm opening width.

- Resected lesion volume: calculated from volumetric segmentation of the target resection region on preoperative MRI (in $\mathrm{cm}^{3}$ ).

- CSF loss via ventricular penetration: determined by whether the ventricular system was entered during lesion resection. The CSF losses through opening the subarachnoid CSF cisterns or performing arachnoid lysis were not considered.

- Presence of brain edema: judged intraoperative by the neurosurgeon observing brain swelling at the time of dural opening, immediately prior to performing the cranial and dural opening scan $\left(\mathrm{MR}_{3}\right)$, and during lesion resection.

Other intraoperative information collected included lesion location (side and lobar sites), total dosage of ce- 


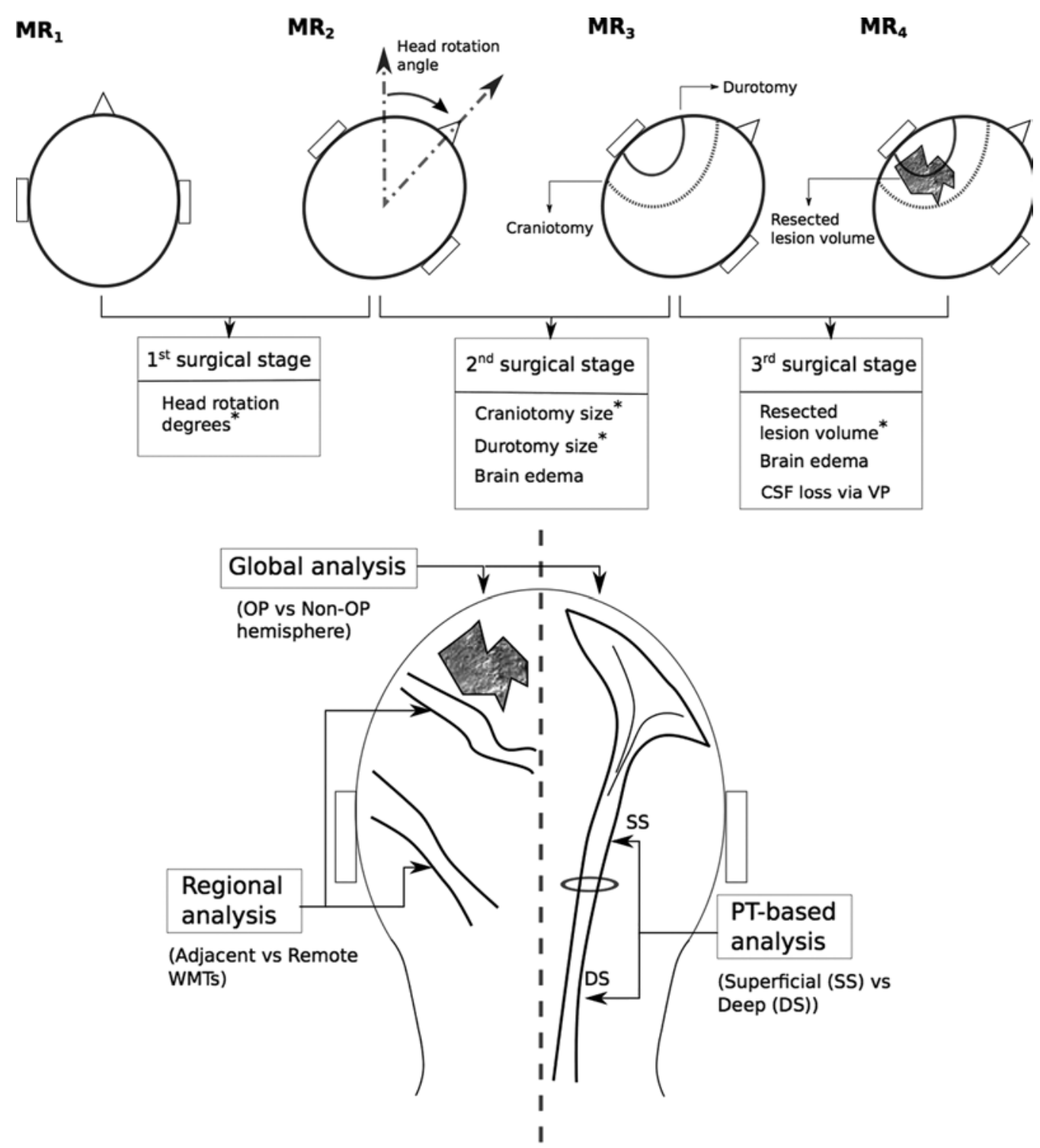

FIG. 2. Illustration of the analysis performed for the intraoperative WMT shift study. The effects of the 6 different IOFs were investigated across 3 pairs of MRI scans obtained over 4 consecutive time points $\left(M R_{1}\right.$ to $\left.M R_{4}\right)$, representing the 3 surgical stages. The

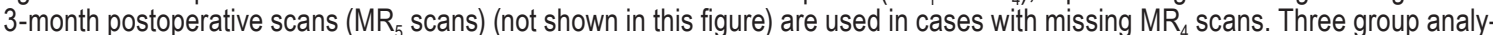
ses were performed for the 4 IOFs marked with asterisks $\left(^{*}\right)$. The remaining 2 IOFs are accounted for in the multiple intraoperative factors regression analyses carried out across the second and the third surgical stages. Non-OP = nonoperative; $\mathrm{OP}=$ operative; $\mathrm{VP}=$ ventricular penetrations.

rebral relaxation agents (diuretics/steroid) used, averaged end-tidal carbon dioxide $\left(\mathrm{ET}-\mathrm{CO}_{2}\right)$ levels prior to all intraoperative MRI scans, the use of a brain retractor, and intraoperative complications, such as intra- or extraaxial hematoma or pneumocephalus.

\section{Tract Shift Evaluation}

WMT position was estimated using the centerline or skeleton of the tract. Tract shift was then quantified by measuring the distance between a pair of centerlines. The distance between a pair of centerlines was defined as the median of the distances between each voxel in one centerline and the closet voxel in the other centerline.

\section{Data and Statistical Analysis}

Linear mixed-effects regression, with patient ID as the random effect, was used to investigate the relationship between regional and global WMT shift and IOFs. Mixedeffects models were used to account for multiple longitudinal measurements obtained from the same individual. Figure 2 summarizes the data analysis scheme used to address both study aims.

WMT shift between $M_{1}$ and $M_{2}$ was used to assess the effect of head rotation, while shift between $\mathrm{MR}_{2}$ and $\mathrm{MR}_{3}$ was used to assess effects of cranial and dural opening size and the presence of brain edema. WMT shift between $\mathrm{MR}_{3}$ and $\mathrm{MR}_{4}$ (or $\mathrm{MR}_{5}$ if $\mathrm{MR}_{4}$ was not available) 
was used to assess the effect of resected lesion volume, CSF loss, and the presence of brain edema.

Each IOF was examined in 3 ways: 1) comparing WMT shift in the operative and the nonoperative hemispheres, 2) comparing shift of WMTs adjacent to and remote to the resected lesion in the operative hemisphere (adjacent defined as those WMTs closer than 1 gyral boundary to the lesion), and 3) comparing shift of the superficial and deep segments of the PT in the operative hemisphere, where the seed ROI at the posterior limb of internal capsule was used to split the tract. The PT was chosen because it has both superficial and deep components.

The durotomy size models were estimated separately for "slit-opened" and "fully opened" durotomies. Group analyses were not possible for the "ventricular penetration" and the "brain edema" factors due to limited dichotomized patient subsets. Thus, these 2 IOFs were only accounted for in the multiple IOFs regression analysis (see statistical analysis below).

Statistical analysis was performed using the R software environment for statistical computing and graphics (R Core Team, 2013; http://www.r-project.org/). Supplementary Document 1 provides details on how to interpret all regression models used in this study. In brief, linear regression analysis accounting for a single IOF was first performed across all 3 pairs of MRI time points. Linear mixed-effects regression analysis accounting for multiple IOFs was then performed across the second and the third pairs of MRI time points (i.e., between $\mathrm{MR}_{2}$ and $\mathrm{MR}_{3}$ and between $\mathrm{MR}_{3}$ and $\mathrm{MR}_{4}$ ). This second step was necessary to dissect the independent effect of each IOF involved across the last 2 pairings of MRI time points. All regression analyses were performed with and without the intracranial volume (ICV) as a covariate.

For each regression model, the interaction between the group (i.e., operative/nonoperative, adjacent/remote, superficial/deep segment) and the designated IOF variable was first examined. If the interaction was not statistically significant, the regression model was tested without the interaction term to examine the main effects of either the group or the IOF variable. Statistics reporting included the regression coefficients, $p$ value, and standard error (SE). A $\mathrm{p} \leq 0.05$ was defined as statistically significant. Effect size was reported by using the marginal $\mathrm{R}^{2}\left(\mathrm{R}_{\text {mar }}^{2}\right)$ and associated probability of superiority (PS). A large effect size was defined as $\mathrm{R}_{\text {mar }}^{2}>0.14$ and PS $>71$; a medium effect size was defined as $\mathrm{R}^{2}{ }_{\text {mar }}>0.05$ and PS $>64$; and a small effect size was defined as $\mathrm{R}_{\text {mar }}^{2}<0.01$ and $\mathrm{PS}<56 .{ }^{13}$

\section{Results}

All 16 patients underwent resection of the epileptogenic zone based on converging preoperative and intraoperative data, mostly entailing lesionectomy. Twelve patients were seizure free at the 3-month postoperative follow-up visit. No patient developed permanent motor and language deficits as a result of surgery. One patient developed an expected homonymous superior quadrantanopia, confirmed by formal visual field perimetry. The resection involved the anterior temporal lobe and AB-OR (Meyer's loop). Two patients demonstrated clinical signs suggestive of a homonymous hemianopia, but visual field perimetry was not possible in these 2 cases due to the patients' young age. In one of these cases, there was an expected visual field deficit due to extensive multifocal corticectomy, involving the parietal and temporal cortices. In the other, the patient had undergone resection of an angular gyrus dysembryoplastic neuroepithelial tumor (DNET), adjacent to the sagittal striatum white matter, containing the OR. The sagittal striatum was intact on the immediate postresection scan $\left(\mathrm{MR}_{4}\right)$ but showed significant atrophic changes on 3-month follow up scan $\left(\mathrm{MR}_{5}\right)$. Proximity of the resection resulting in compromised vascular supply to the sagittal striatum was the likely cause.

The pathology of the resected epileptogenic lesions was focal cortical dysplasia $(n=4 ; 1$ patient also had a developmental venous anomaly), tuberous sclerosis complex (n $=3)$, DNET $(\mathrm{n}=5)$, chronic encephalitis $(\mathrm{n}=1)$, cerebral gliosis secondary to a previous tumor resection $(n=2)$, and nonspecific findings $(\mathrm{n}=1)$.

The intraoperative information is summarized in Table 1. The surgeries included a wide range of craniotomy and durotomy sizes and various lesion sites and sizes involving each cerebral hemisphere. The majority of the surgeries (10 of 16 cases) were performed with the patient's head rotated $90^{\circ}$ laterally. Ventricular penetration occurred in 2 cases. Seven patients had brain edema before and during lesion resection. While the average ET- $\mathrm{CO}_{2}$ level at the time of MRI scanning and average intravenous steroid doses were similar in all cases, the patients' head positions and transient elevations of ET- $\mathrm{CO}_{2}$ levels contributed to the brain edema initially observed in these cases. Combined maneuvers of brief hyperventilation, head elevation, CSF release via piotomy and arachnoid lysis, and slit durotomy were able to partially reverse the brain edema and allow for safe intraoperative imaging. Brain retractors were not required for resection in any case. None of the patients had significant intracerebral hemorrhage or extraaxial collection/pneumocephalus.

$\mathrm{MR}_{1}, \mathrm{MR}_{2}$, and $\mathrm{MR}_{3}$ scans were acquired in all patients. Five $M_{1}$ scans were acquired under general anesthesia. Four of the acquired $\mathrm{MR}_{1}$ scans were excluded because they were acquired on a collocated 1.5-T MRI scanner through error. $\mathrm{MR}_{4}$ scans were acquired in 13 of 16 patients. Three $\mathrm{MR}_{4}$ scans were not performed due to safety and logistic issues related to the long surgical time. Two $\mathrm{MR}_{4}$ scans were excluded from further analysis. One had poor image quality due to significant EPI susceptibility distortion and head motion artifact. The other had incomplete acquisition relating to MRI hardware malfunction. In the 5 patients without usable $\mathrm{MR}_{4}$ scans, $\mathrm{MR}_{5}$ scans were used instead for relevant data analysis. Two of these $5 \mathrm{MR}_{5}$ scans were acquired under general anesthesia. Visual inspection of the HARDI diffusion data revealed negligible image distortions related to eddy current and motion artifacts.

MRI data from the following pairs of scans were available for analyses: 12 pairs of $\mathrm{MR}_{1}$ and $\mathrm{MR}_{2}$ scans; 16 pairs of $\mathrm{MR}_{2}$ and $\mathrm{MR}_{3}$ scans; 11 pairs of $\mathrm{MR}_{3}$ and $\mathrm{MR}_{4}$ scans; and 5 pairs of $\mathrm{MR}_{3}$ and $\mathrm{MR}_{5}$ scans.

The average combined time spent for preparing the operating room, additional general anesthesia, and intraoperative MRI scanning time per case was 142 minutes (range 

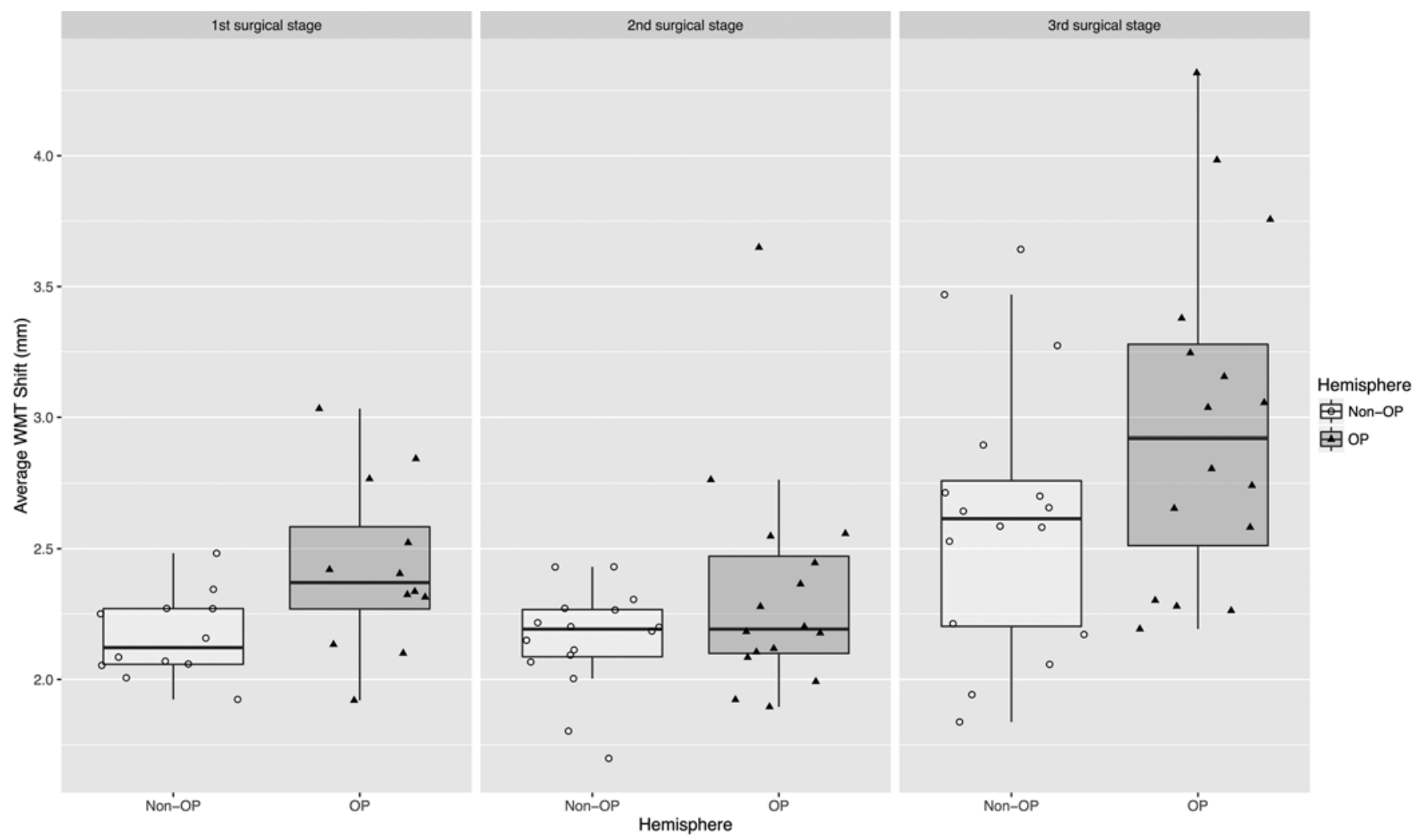

FIG. 3. Box and whisker plots showing the average WMT shift in both operative (OP) and nonoperative (Non-OP) hemispheres across each pair of MRI scans, representing the 3 surgical stages. The plots demonstrate similar degrees of WMT shift in both hemispheres. Greater degrees and ranges of WMT shift are observed across the third surgical stage.

105-190 minutes; on average 53 minutes per scan session, range 44-62 minutes).

\section{Average WMT Shift in Both Cerebral Hemispheres}

The results of average WMT shift in both cerebral hemispheres across the 3 surgical stages are summarized in Fig. 3.

The median of average WMT shifts resulting from change in head position $\left(\mathrm{MR}_{1}\right.$ and $\left.\mathrm{MR}_{2}, \mathrm{n}=12\right)$ was 2.37 $\mathrm{mm}$ (range 1.92-3.03 $\mathrm{mm}$ ) for the operative hemisphere and $2.12 \mathrm{~mm}$ (range $1.92-2.48 \mathrm{~mm}$ ) for the nonoperative hemisphere.

The median of average WMT shifts after cranial and dural opening and in the presence of brain edema $\left(\mathrm{MR}_{2}\right.$ and $\mathrm{MR}_{3}, \mathrm{n}=16$ ) was $2.19 \mathrm{~mm}$ (range $1.90-3.65 \mathrm{~mm}$ ) in the operative hemisphere and $2.19 \mathrm{~mm}$ (range 1.70-2.43 $\mathrm{mm}$ ) in the nonoperative hemisphere.

The median of average WMT shifts after lesion resection and in the presence of brain edema and ventricular penetration $\left(\mathrm{MR}_{3}\right.$ and $\mathrm{MR}_{4}$ [or $\mathrm{MR}_{5}$ ], $\mathrm{n}=16$ ) was 2.92 $\mathrm{mm}$ (range 2.19-4.32 $\mathrm{mm}$ ) for the operative hemisphere and $2.62 \mathrm{~mm}$ (range $1.84-3.64 \mathrm{~mm}$ ) for the nonoperative hemisphere.

\section{Single-IOF Regression Analysis}

ICV was not statistically significant $(\mathrm{p}>0.2)$ when included as a covariate in any regression model, and thus regression analyses were reported without including ICV.
A summary of the IOF regression analysis results is provided in tabular form in Fig. 4. Only the statistically significant results are shown in the table (please refer to Supplementary Tables 2-4 for all analysis statistics). A significant interaction between IOF and a group variable is indicated by the coefficient " $(\beta 3-\beta 2)$ ". A significant main effect for the IOF variable (in a model without interaction) is indicated by $\beta 2$, and a significant main effect of the group variable is indicated by " $(\beta 1-\beta 0)$ ".

Large effect sizes were observed in all reported statistics showing significant findings $\left(\mathrm{R}_{\text {mar }}^{2}=0.15-0.76\right.$; $\mathrm{PS}=$ 72-99).

A significant interaction was identified between the "fully opened" durotomy size and the hemisphere, with the WMT shift increasing more rapidly with the durotomy size in the operative rather than in the nonoperative hemisphere (statistics are provided in the navy blue section in Fig. 4; Fig. 5 shows the regression plot). No significant interactions or main effects were observed in the "slitopened" durotomy model analysis $(p>0.2)$. A significant interaction was also identified between the resected lesion volume and the hemisphere, with the WMT shift increasing more rapidly with the increase in resected lesion volume in the operative rather than in the nonoperative hemisphere (statistics in the green section in Fig. 4). No other significant interaction effects were observed.

The WMT shift increased with the durotomy size but did not depend on the lesion proximity (yellow section in 


\begin{tabular}{|c|c|c|c|c|c|c|}
\hline \multirow{3}{*}{ IOFs } & \multicolumn{6}{|c|}{ Group Variables } \\
\hline & \multicolumn{2}{|c|}{ Op vs Non-Op } & \multicolumn{2}{|c|}{ Adjacent vs Remote WMTs } & \multicolumn{2}{|c|}{ Superficial vs Deep Segment of PT } \\
\hline & $\begin{array}{c}\text { With } \\
\text { Interaction }\end{array}$ & $\begin{array}{l}\text { Without } \\
\text { Interaction }\end{array}$ & $\begin{array}{c}\text { With } \\
\text { Interaction }\end{array}$ & $\begin{array}{c}\text { Without } \\
\text { Interaction }\end{array}$ & $\begin{array}{c}\text { With } \\
\text { Interaction }\end{array}$ & $\begin{array}{l}\text { Without } \\
\text { Interaction }\end{array}$ \\
\hline Head rotation $\left({ }^{\circ}\right)$ & NS & $\begin{aligned}(\beta 1-\beta 0) \pm S E & =0.26 \pm 0.08 \\
\left(p=0.007 ; R_{\operatorname{mar}}^{2}\right. & =0.20 ; P S=76)\end{aligned}$ & NS & NS & NS & $\begin{aligned}(\beta I-\beta 0) \pm S E & =-0.69 \pm 0.32 \\
\left(p=0.05 ; R_{\operatorname{mar}}^{2}\right. & =0.14 ; P S=71)\end{aligned}$ \\
\hline Craniotomy size & NS & NS & NS & NS & NS & $\begin{aligned}(\beta 1-\beta 0) \pm \mathrm{SE} & =1.09 \pm 0.23 \\
\left(\mathrm{p}<0.001 ; \mathrm{R}_{\operatorname{mar}}^{2}\right. & =0.36 ; \mathrm{PS}=86)\end{aligned}$ \\
\hline Durotomy size & $\begin{array}{c}(\beta 3-\beta 2) \pm \mathrm{SE}=0.02 \pm 0.003 \\
\left(\mathrm{p}<0.001 ; \mathrm{R}_{\operatorname{mar}}^{2}=0.76 ; \mathrm{PS}=99\right)\end{array}$ & NR & NS & $\begin{array}{c}\beta 2 \pm \mathrm{SE}=0.03 \pm 0.006 \\
\left(p=0.003 ; R_{\operatorname{mar}}^{2}=0.56 ; \mathrm{PS}=95\right)\end{array}$ & NS & $\begin{array}{c}\beta 2 \pm S E=0.03 \pm 0.01 \\
\left(p=0.05 ; R_{\text {mar }}^{2}=0.54 ; P S=93\right) \\
(\beta 1-\beta 0) \pm S E=1.23 \pm 0.30 \\
(p=0.004)\end{array}$ \\
\hline Resected lesion vol & $\begin{array}{c}(\beta 3-\beta 2) \pm S E=0.009 \pm 0.002 \\
\left(p<0.001 ; R_{\operatorname{mar}}^{2}=0.36 ; P S=86\right)\end{array}$ & NR & NS & $\begin{array}{c}\beta 2 \pm \mathrm{SE}=0.02 \pm 0.007 \\
\left(\mathrm{p}=0.04 ; \mathrm{R}_{\operatorname{mar}}^{2}=0.29 ; \mathrm{PS}=81\right) \\
(\beta 1-\beta 0) \pm \mathrm{SE}=-0.46 \pm 0.16 \\
(\mathrm{p}=0.01)\end{array}$ & NS & $\begin{array}{c}\beta 2 \pm \mathrm{SE}=0.02 \pm 0.01 \\
\left(\mathrm{p}=0.009 ; \mathrm{R}_{\mathrm{mar}}^{2}=0.27 ; \mathrm{PS}=80\right)\end{array}$ \\
\hline
\end{tabular}

FIG. 4. Summary of single-IOF regression analysis results. The statistically significant results are shown with colored background. The colors correspond to the descriptions in the Results section; $\beta 2$, ( $\beta 1-\beta 0)$, and ( $\beta 3-\beta 2)$ are the regression coefficients for the analyses. The patients with "slit-opened" durotomies $(n=7)$ were excluded from the durotomy size analysis. NR = nonrelevant statistics (given significant interaction is observed); NS = nonsignificant (results); op, non-op = operative, non-operative. Figure is available in color online only.

Fig. $4 ; \beta 2 \pm \mathrm{SE}=0.03 \pm 0.006, \mathrm{p}=0.003, \mathrm{R}_{\text {mar }}^{2}=0.56$, $\mathrm{PS}$ =95). The PT shift increased with the durotomy size, and the increase was greater in its superficial segment than in the deeper segment (light green section in Fig. $4 ; \beta 2 \pm$ SE $=0.03 \pm 0.01, \mathrm{p}=0.05 ;[\beta 1-\beta 0] \pm \mathrm{SE}=1.23 \pm 0.30, \mathrm{p}=$ $0.004, \mathrm{R}_{\text {mar }}^{2}=0.54 ; \mathrm{PS}=93$ ). The WMT shift increased with the resected lesion volume, and the increase was greater in the tracts adjacent rather than remote to the lesion (cyan section in Fig. 4; $\beta 2 \pm \mathrm{SE}=0.02 \pm 0.007, \mathrm{p}=$ $0.04 ;[\beta 1-\beta 0] \pm \mathrm{SE}=-0.46 \pm 0.16, \mathrm{p}=0.01, \mathrm{R}_{\mathrm{mar}}{ }^{2}=0.29, \mathrm{PS}$ $=81$ ). However, the WMT shift increase with the resected lesion volume was independent of the PT segment depth (pink section in Fig. 4; $\beta 2 \pm \mathrm{SE}=0.02 \pm 0.01, \mathrm{p}=0.009$, $\mathrm{R}_{\text {mar }}^{2}=0.27, \mathrm{PS}=80$ ).

The WMT shift increase was greater in the operative hemisphere independent of the degrees of head rotation (red section in Fig. 4; $[\beta 1-\beta 0] \pm \mathrm{SE}=0.26 \pm 0.08, \mathrm{p}=$ $0.007, \mathrm{R}^{2}$ mar $=0.20, \mathrm{PS}=76$ ). The WMT shift increase was greater in the superficial PT segment independent of the head rotation degrees (orange section in Fig. 4; $[\beta 1-\beta 0] \pm$ $\left.\mathrm{SE}=-0.69 \pm 0.32, \mathrm{p}=0.05, \mathrm{R}_{\text {mar }}^{2}=0.14, \mathrm{PS}=71\right)$, and the craniotomy size (purple section in Fig. 4 ; $[\beta 1-\beta 0] \pm \mathrm{SE}=$ $1.09 \pm 0.23, \mathrm{p}<0.001, \mathrm{R}_{\text {mar }}^{2}=0.36, \mathrm{PS}=86$ ).

TABLE 2. Multiple intraoperative factor regression analysis across $M R_{2}$ and $M R_{3}$, the second surgical stage

\begin{tabular}{|c|c|c|}
\hline \multirow[b]{2}{*}{ Independent Variable } & \multicolumn{2}{|c|}{$\begin{array}{c}\mathrm{RA} \text { w/ Interaction } \\
\left(\mathrm{R}^{2}{ }_{\mathrm{mar}}=0.55, \mathrm{PS}=94\right)\end{array}$} \\
\hline & $\beta \pm S E$ & $\mathrm{p}$ Value \\
\hline$\beta 0$ (baseline intercept) & $1.86 \pm 0.17$ & $<0.001$ \\
\hline$\beta 1$ (op side) & $-0.13 \pm 0.08$ & 0.11 \\
\hline$\beta 2$ (durotomy size) & $0.007 \pm 0.005$ & 0.19 \\
\hline$\beta 4$ (presence of brain edema) & $0.31 \pm 0.16$ & 0.08 \\
\hline$\beta 5$ (craniotomy size) & $0.0005 \pm 0.003$ & 0.85 \\
\hline ( $\beta 3-\beta 2)$ (durotomy size:op side) interaction & $0.015 \pm 0.003$ & $<0.001$ \\
\hline
\end{tabular}

$\mathrm{RA}=$ regression analysis.

Boldface type indicates statistical significance $(p \leq 0.05)$. $\beta 0-\beta 5$ are the regression coefficients of the independent variables used for the analysis.
The regression coefficient $\beta 0$ intercepts of all group main effect analyses performed were nonzero (all $\mathrm{p} \leq$ $0.005)$. This implied WMT shifts occurring in the nonoperative hemisphere, in the WMTs remote to the lesion, and in the deep segment of the PT in the operative hemisphere were all significantly greater than zero.

\section{Multiple-IOF Regression Analysis Results}

The analysis was performed using the hemispheric sides as the group variable. The results are summarized in Table 2 (across $\mathrm{MR}_{2}$ and $\mathrm{MR}_{3}$ ) and Table 3 (across $\mathrm{MR}_{3}$ and $\mathrm{MR}_{4}$ [or $\left.\mathrm{MR}_{5}\right]$ ). These analyses include additional group variables for edema and ventricular penetration.

\section{Across $\mathrm{MR}_{2}$ and $\mathrm{MR}_{3}$}

A significant interaction was detected between the durotomy size and the operative hemisphere $(\mathrm{p}<0.001)$ across $\mathrm{MR}_{2}$ and $\mathrm{MR}_{3}$ - the second surgical stage. This meant that increased durotomy size had resulted in significantly greater WMT shifts in the operative hemisphere than in the nonoperative hemisphere, independent of the

TABLE 3. Multiple intraoperative factor regression analysis across $M R_{3}$ and $M R_{4}$ (or $M R_{5}$ ), the third surgical stage

\begin{tabular}{|c|c|c|}
\hline \multirow[b]{2}{*}{ Independent Variable } & \multicolumn{2}{|c|}{$\begin{array}{c}\text { RA w/ Interaction } \\
\left(R_{\text {mar }}^{2}=0.36, P S=86\right)\end{array}$} \\
\hline & $\beta \pm S E$ & $\mathrm{p}$ Value \\
\hline$\beta 0$ (baseline intercept) & $2.65 \pm 0.20$ & $<0.001$ \\
\hline$\beta 1$ (op side) & $0.15 \pm 0.09$ & 0.14 \\
\hline$\beta 2$ (resected lesion vol) & $0.003 \pm 0.003$ & 0.33 \\
\hline$\beta 4$ (presence of brain edema) & $-0.25 \pm 0.28$ & 0.39 \\
\hline$\beta 5$ (CSF loss via ventricular penetration) & $-0.09 \pm 0.40$ & 0.83 \\
\hline $\begin{array}{l}\text { ( } \beta 3-\beta 2) \text { (resected lesion vol: op side) } \\
\text { interaction }\end{array}$ & $0.009 \pm 0.002$ & $<0.001$ \\
\hline
\end{tabular}

Boldface type indicates statistical significance $(p \leq 0.05)$. $\beta 0-\beta 5$ are the regression coefficients of the independent variables used for the analysis. 
TABLE 4. Results summary with respect to the study hypotheses

\begin{tabular}{cl}
\multicolumn{1}{c}{ Variables or Factor } & \multicolumn{1}{c}{$\begin{array}{c}\text { Does the result support } \\
\text { the hypothesis? }\end{array}$} \\
\hline $\begin{array}{c}\text { Result summary by group variables } \\
\text { Op vs non-op hemisphere }\end{array}$ & Yes (across all 3 surgical stages) \\
\hline Adjacent vs remote WMTs & Yes (across 3rd surgical stage) \\
\hline $\begin{array}{c}\text { Superficial vs deep PT segment } \\
\text { Result summary by intraoperative } \\
\text { factors }\end{array}$ & Yes (across 2nd surgical stage) \\
\hline Head rotation (degrees) & No \\
\hline Craniotomy size & No \\
\hline Durotomy size & Yes* (across 2nd surgical stage) \\
\hline Resected lesion volume & Yes* (across 3rd surgical stage) \\
\hline $\begin{array}{c}\text { CSF loss via ventricular } \\
\text { penetration }\end{array}$ & No \\
\hline Presence of brain edema & Trend (across 2nd surgical stage) \\
\hline
\end{tabular}

* Significant independent effect by multiple intraoperative factor regression analysis.

other 2 IOFs. The presence of brain edema was only marginally significant $(p=0.08)$. The craniotomy size was not a significant independent factor affecting the average WMT shift in the operative hemisphere $(\mathrm{p}=0.85)$. A large effect size was observed for this analysis $\left(\mathrm{R}^{2}\right.$ mar $=0.55$, PS $=94)$.

\section{Across $\mathrm{MR}_{3}$ and $\mathrm{MR}_{4}$ (or $\mathrm{MR}_{5}$ )}

A significant interaction was detected between the resected lesion volume and the operative hemisphere $(p<$ 0.001; large effect size: $\mathrm{R}_{\text {mar }}^{2}=0.36, \mathrm{PS}=86$ ) across $\mathrm{MR}_{3}$ and $\mathrm{MR}_{4}$ (or $\mathrm{MR}_{5}$ ) - the third surgical stage. This meant that increased resected lesion volume resulted in significantly greater WMT shifts in the operative hemisphere than in the nonoperative hemisphere, independent of the other 2 IOFs.

The analysis also suggested that neither the presence of brain edema nor ventricular penetration was a significant independent variable affecting the average WMT shift across $\mathrm{MR}_{3}$ and $\mathrm{MR}_{4}\left(\right.$ or $\mathrm{MR}_{5}$ ).

\section{Direction of WMT Shift}

The direction of WMT shift was variable and independent of the cortical surface brain shift. It was influenced by multiple interactive IOFs, such as the head position (i.e., gravity), presence of brain edema, ventricular penetration, and following lesion resection. Figures 6 and 7 and Video 1 show selected WMT shift images from one patient (Case 2), highlighting these pertinent findings.

VIDEO 1. Video clip demonstrating a 3D rotating file of Fig. 6.

Copyright Joseph Yuan-Mou Yang. Published with permission. Click here to view.

\section{Discussion}

In this study, we characterized WMT shift during the

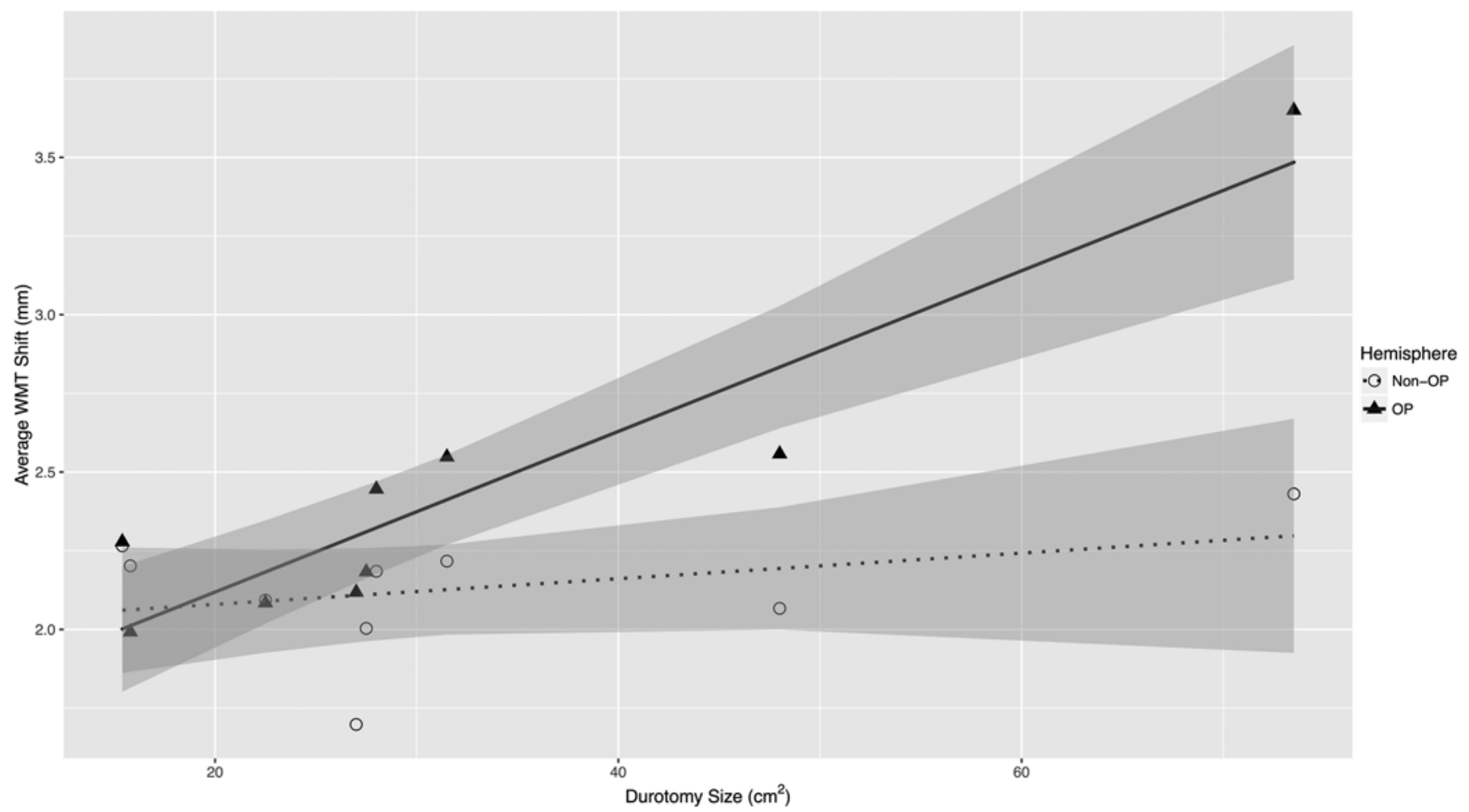

FIG. 5. Average WMT shift examined by interaction analysis between "fully opened" durotomy size and the hemispheric group variable, across $\mathrm{MR}_{2}$ and $\mathrm{MR}_{3}$ scans (the second surgical stage). The gray-shaded areas on either side of the regression lines represent the $95 \%$ confidence intervals. The plot shows that significant interaction existed between the durotomy size and the hemispheric group variables. It implies that increased durotomy size is associated with greater degree of average WMT shift in the operative hemisphere (OP) than the nonoperative (Non-OP) hemisphere. 

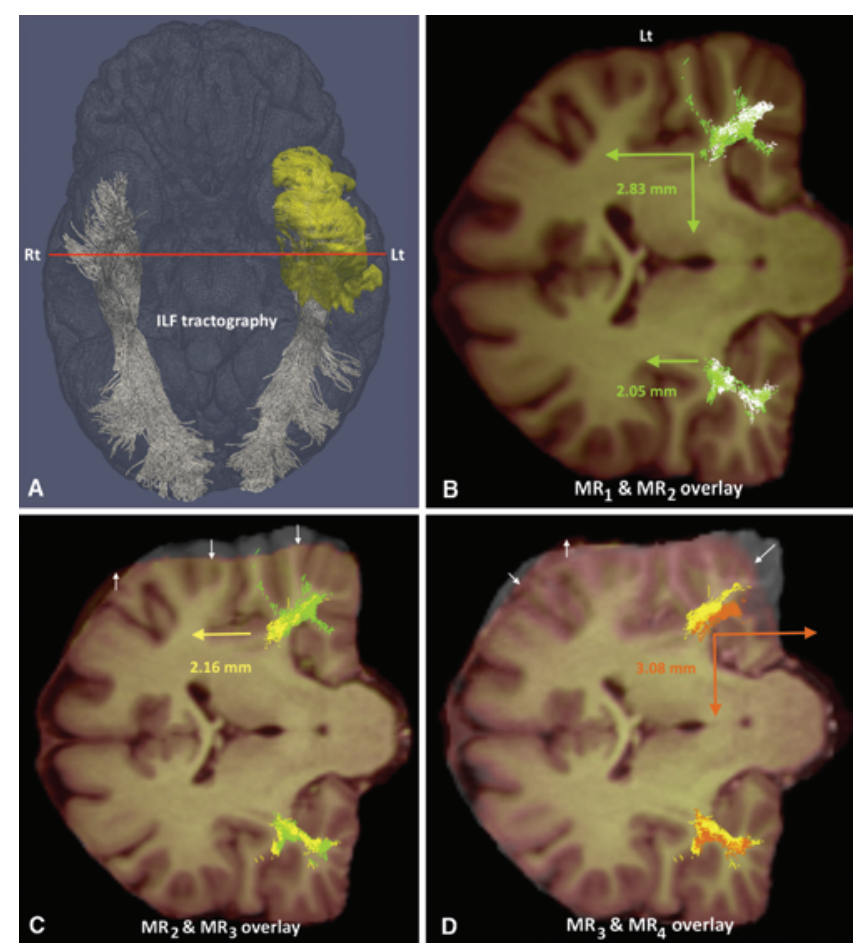

FIG. 6. Case 2. Overlaying consecutive perioperative MR images and ILF tractography showing the differences in the progressive cortical surface and subcortical WMT shifts over the course of the surgery. This 13-year-old boy's operation involved resection of a $16.5-\mathrm{cm}^{3}$ left anterior and mesial temporal focal cortical dysplasia (lesion indicated by yellow area in A). He underwent a left temporal craniotomy with $90^{\circ}$ lateral head position. A 55.3- $\mathrm{cm}^{2}$ craniotomy and a $48.0-\mathrm{cm}^{2}$ durotomy were performed. The operation was uneventful. The ventricular system was not entered, and there was no visible brain edema. A: 3D rendered brain image reconstructed using the preoperative MRI, showing ILF tractography in both hemispheres and the targeted resecting lesion. The red line represents the coronal level of MR images displayed in B-D. B-D: Overlapped images of temporal stem portions of ILF from consecutive MRI pairs are displayed on top of the overlapped anatomical T1-weighted MR images. The ILFs from the 4 consecutive MRI scans are labeled with different colors (white for $\mathrm{MR}_{1}$, green for $\mathrm{MR}_{2}$, yellow for $\mathrm{MR}_{3}$, and orange for $\mathrm{MR}_{4}$ ). The coronal MR images are displayed in radiological convention with lateral orientation, simulating the surgical position. White arrows mark the direction of cortical surface brain shift. Colored arrows mark the directions of the ILF shift across each pair of MR images. The length of the arrows represents magnitude of the tract shift. The images demonstrate the directions of ILF shift occurred are variable and independent to the shift observed at the cortical surface. Figure is available in color online only.

course of epilepsy surgery by means of pairwise comparison of MRI scans performed over 5 perioperative time points. Eleven different association and projection WMT/ WMT segments were reconstructed using CSD modeling of HARDI diffusion data and probabilistic tractography. The pairwise MRI comparisons enabled both the etiology and the magnitude of WMT shift to be studied across 3 consecutive surgical stages. We then examined and dissociated the impacts of 6 different IOFs on the tract shift, from a global hemispheric to a regional tract-based scale.

The study demonstrated a median value of average WMT shift occurring in the operative hemisphere of about

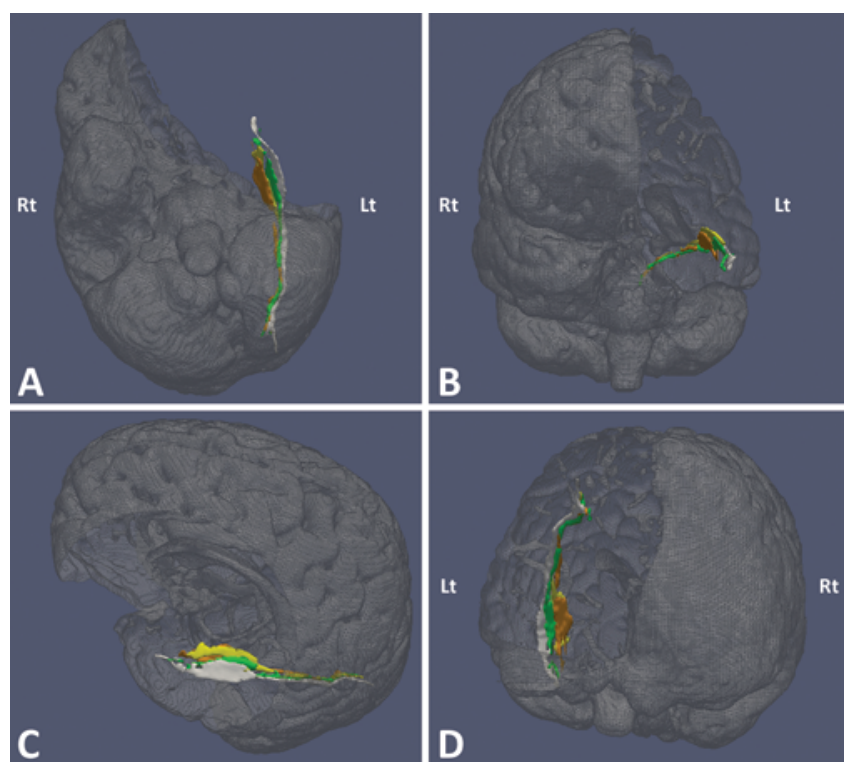

FIG. 7. Case 2. 3D reconstructions of ILF tractography centerlines over 4 consecutive MRI scans displayed on partially cut-away brain images. The color schemes of the centerlines reflect the 4 MRI time points (white for $\mathrm{MR}_{1}$, green for $\mathrm{MR}_{2}$, yellow for $\mathrm{MR}_{3}$, and orange for $\mathrm{MR}_{4}$ ). The selected viewpoints demonstrate the 3D changes in ILF shift orientations, progressively over the surgical course. A: Inferior view. B: Anterior view. C: Lateral view. D: Posteroinferior view. Figure is available in color online only.

$2.5 \mathrm{~mm}$ for each of the 3 surgical stages. This equates to a total maximum shift of about $7.5 \mathrm{~mm}$, if shift at each stage is in the same direction. Table 4 summarizes the results against the study hypotheses by the group variables and by the IOFs.

WMT shift was greater in the operative hemisphere (across all surgical stages), in the WMTs closer to the lesion (across the third surgical stage), and in the superficial rather than deeper segment of the PT (across the second surgical stage). Greater WMT shift occurred with increased durotomy size and increased resected lesion volume, independent of the effects of other IOFs. The presence of brain edema was only a marginally significant IOF. The hypothesis of greater WMT shift with increased craniotomy size is not supported. The results do not support the hypotheses of greater WMT shift in context of increased head rotation (as measured in degrees) and with increased CSF loss via ventricular penetration.

Direct outcome comparisons with other studies are constrained by differences in study design, MRI hardware, and methodological approaches of tractography and analysis of WMT shift. Our study is the first to address WMT shift in pediatric patients undergoing epilepsy surgery, incorporate data from a high-field intraoperative MRI scanner, and address WMT shift across multiple surgical stages.

The average WMT shift demonstrated in the operative hemisphere in our study is consistent with other reports. ${ }^{31,41-43,49}$ Nimsky et al. ${ }^{41}$ in 2005 reported a maximum 15-mm (either inward or outward) intraoperative WMT shift following resective surgery performed in 38 mixed adult and pediatric brain tumor and temporal lobe epilep- 
sy cases. Maesawa et al. ${ }^{31}$ reported an average PT shift of $6.65 \mathrm{~mm}$ in 28 mixed adult and pediatric brain tumor cases. Romano et al. ${ }^{49}$ investigated intraoperative PT shift in 20 adult brain tumor cases, with additional intraoperative MRI performed after dural opening, and reported a maximum 7.50-mm (either inward or outward) PT shift after dural opening. This is similar to our finding of total WMT shift for the first and the second surgical stages $(6.68 \mathrm{~mm})$. Further, Romano et al. also reported a maximum 9.70-mm inward to $11.00-\mathrm{mm}$ outward PT shift after tumor resection. This is also consistent with our finding $(11.00 \mathrm{~mm})$. Despite the differences in the studied pathology, similar resection lesion volumes were reported in our study (26.4 $\mathrm{cm}^{3}$, range $3.9-163.3 \mathrm{~cm}^{3}$ ) and the other 2 studies reporting the lesion volumes (range 4.0-149 $\mathrm{cm}^{3}$ ). ${ }^{42,49}$

The finding of WMT shift occurring in the nonoperative hemisphere is unique to our study. Other studies assessed image registration accuracy based on the assumption that negligible brain shift took place in the nonoperative hemisphere. This may not be true, because brain shift is a dynamic biomechanical process. ${ }^{33,37}$ The etiology of this process is multifactorial and additive. The gravitational effect predominantly drives the tract shift during the initial surgical stage. The lesser tract shift that we observed in the nonoperative hemisphere supports this claim, given that the operative field is always positioned uppermost. With increased surgical time, progressively more tract shift occurred in the nonoperative hemisphere as result of the body's own hemodynamic and CSF volume regulation following cranial and dural opening and lesion resection. These effects are also in part due to the complex interplay between administered anesthetic and diuretic agents, intravenous fluid therapies, and the ventilation/ET-CO $-\mathrm{Cari}_{2}$ ability occurring over the surgical time course.

Our findings showing lack of significant effects of several IOFs on the WMT shift are somewhat counterintuitive and inconsistent with other reports.37,39,42,49 The lack of effect for the head rotation degrees and the degree of CSF loss could be due to the sampling bias and the method used to define the IOF. Thirteen of the 16 surgeries were performed in the lateral $\left(90^{\circ}\right.$ head rotation, $\left.\mathrm{n}=10\right)$ and oblique lateral $\left(120^{\circ}\right.$ head rotation, $\left.n=3\right)$ positions. Analysis of ventricular penetration as a single IOF was not possible due to the small sample size $(n=2)$. We analyzed CSF loss by ventricular penetration, and ignored the potentially significant loss through opening the subarachnoid CSF cisterns. There is no good mechanism to accurately quantify the loss of CSF that is not related to ventricular puncture. The effect of CSF loss may be masked by the effect of lesion resection across the last surgical stage. The lack of effect for craniotomy size is related to the discordance between the cranial and dural opening size in our study cohort. In 7 of 16 patients, only slit durotomy was performed prior to the second intraoperative $\left(\mathrm{MR}_{3}\right)$ scan, due to associated brain edema. The dura mater was purposely not opened in full to prevent dangerous brain herniation. Other studies do not make such a distinction. ${ }^{49}$ The marginal effect of the presence of brain edema in this study is attributable to the stabilization of edema prior to scanning and the small number of patients with edema.

The findings of this study have several obvious clinical implications. Intraoperative WMT shift is a dynamic process that is more difficult to observe than cortical surface shift. Visualization of cortical surface shift can be guided by the surface gyral and vascular anatomy. ${ }^{39,49}$ Confirmation of the intraoperative WMT position is necessary through shift-compensated tractography reconstruction.

Notable WMT shift can occur following cranial and dural openings, prior to lesion resection (average shift $2.19 \mathrm{~mm}$, range $1.90-3.65 \mathrm{~mm}$ ), and durotomy size and the presence of brain edema are the predominant IOFs influencing the WMT shift at this stage. This highlights the necessity of updating image-guided neuronavigation following dural opening through intraoperative MRI scan acquisition.

The surgical stage-wise breakdown of WMT shift helps with refining the proposed safety resection margin. Conventional evidence comes from direct subcortical electrical stimulation studies validating WMT position proposed by the preoperative diffusion tractography. ${ }^{8,26,34}$ The difference of the functional and structural position is typically around $10.00 \mathrm{~mm}$, representing combined electrical stimulation errors, tracking errors and errors encountered with image registration, and errors from intraoperative brain shift. Our results show that about one-half to twothirds of this previously reported safety margin can be attributed to the intraoperative WMT shift prior to lesion resection, suggesting a more refined safety resection margin of $5.00 \mathrm{~mm}$. Incidentally, this is similar to the "worst case scenario" proposed by Nimsky et al. ${ }^{40}$ when intraoperative WMT shift is not considered.

It is important to bear in mind that the purpose of this study was to evaluate the potential clinical value of advanced tractography measures. Tractography images were retrospectively reconstructed from the HARDI diffusion data and were not used to guide intraoperative neuronavigation. Current navigation systems do not allow for image recalibration incorporating real-time HARDI tractography images. Knowledge acquired from this study could assist with the development of computer simulation models in predicting and correcting registration errors caused by intraoperative brain shift when using preoperative brain images for neuronavigation guidance. ${ }^{24,45,48,51}$ It remains unclear how much and what frequency of intraoperative MRI data are sufficient for these simulation models, given the complexity of brain and WMT shifts occurring in different surgical scenarios and in different procedures. ${ }^{19}$

Interpretation of the findings from this study is limited by the small sample size and heterogeneous epilepsy characteristics and pathologies. We note that large effect sizes were reported from the majority of regression analyses. Recent seizures ${ }^{9,58,59}$ and developmental pathologies with disorganized WMTs $2,15,16,20,27,50$ could contribute to the individual differences in brain diffusion data, thus inadvertently affecting the accuracy of tractography reconstructions. Brain edema was subjectively defined in this study without quantification, and its impact on WMT shifts was partially compensated for, prior to acquisition of the intraoperative images. We used the term "brain edema" as a descriptor of brain swelling that occurred at the time of dural opening. This was multifactorial, being potentially 
due to combinations of perilesional edema, hemodynamic and ventilation factors, and possible seizure activity. The findings with regard to impact of degrees of head rotation and CSF loss on WMT shift require further clarification. Our quantitative analysis of the WMT shifts did not fully address the differences in shift direction between surgical stages. Figure 6 shows that the trajectories of the tract shift during surgery can be complex. Internal inconsistency associated with MRI hardware and scanning noises inherently limits the image registration and tractography accuracy that can be achieved in this study. Although negligible on visual inspection, motion artifacts occur with head movement, and with cardiac/CSF pulsation. Subtle image distortions occur with magnetic field and gradient field inhomogeneity. Radiofrequency noise and signal attenuation occur relating to the surface coil distance. Interscanner variability exists between processing of a conventional diagnostic and an intraoperative MRI scanner. Manual segmentation of the diffusion tractography introduces operator-dependent bias. Aligning a preoperatively acquired scan $\left(\mathrm{MR}_{1}\right)$ and an intraoperatively acquired scan $\left(\mathrm{MR}_{2}\right)$ negates the influence of developmental brain growth. Using $\mathrm{MR}_{5}$ to replace the missing $\mathrm{MR}_{4}$ data negates the WMT shift relating to anatomical restoration and development that may occur postoperatively.

Future directions arising from this study include addressing the effect on WMT shift of the location and pathology-related tissue characteristics in a larger clinical sample. Investigations of WMT shift relating to pharmacological and anesthetic interventions would be of interest but would require controlled manipulation of parameters affecting intracerebral pressure, more appropriately performed in brain phantom or animal models. Practical challenges remain for integration of neuronavigation software capable of processing HARDI diffusion data and performing probabilistic tractography in real-time during surgery. This would involve computational upgrades to improve efficiency of diffusion data acquisition using the intraoperative MRI scan ${ }^{11,35,46}$ and tractography processing using either an automated ${ }^{17,28}$ or a semi-automated, manual interactive method. ${ }^{14}$

\section{Conclusions}

Intraoperative WMT shift is a progressive, dynamic process affecting the whole brain and is influenced by multiple IOFs. This study demonstrates greater WMT shift in the operative hemisphere, in the WMTs adjacent to the surgical lesion, and in the superficial segment of the PT. Noticeable WMT shifts can occur following dural opening, prior to lesion resection. The durotomy size and the resection size are significant independent IOFs affecting the WMT shift. The presence of brain edema is a marginally significant IOF. Application of the tractography techniques and the knowledge acquired from this study may help compensate for intraoperative WMT shift and improve neuronavigation accuracy using either preoperative or intraoperative MRI data.

\section{Acknowledgments}

We thank the operating theater nurses, anesthetic staff, the MRI technologists involved in MRI data acquisition, and the families and children who participated in this study. We thank Dr. Christopher L. Adamson, PhD, for technical support.

This research was conducted within the Department of Neurosurgery, Royal Children's Hospital, and the Developmental Imaging research group, Murdoch Childrens Research Institute at the Melbourne Children's MRI Centre, Melbourne, Victoria. It was supported by the Royal Children's Hospital Foundation (RCH1000 to Dr. Yang), Australia's National Health and Medical Research Council (Postgraduate Scholarship 1039160 to Dr. Yang), Murdoch Childrens Research Institute, The University of Melbourne Department of Paediatrics, and the Victorian Government's Operational Infrastructure Support Program.

\section{References}

1. Alexander DC, Barker GJ: Optimal imaging parameters for fiber-orientation estimation in diffusion MRI. Neuroimage 27:357-367, 2005

2. Assaf BA, Mohamed FB, Abou-Khaled KJ, Williams JM, Yazeji MS, Haselgrove J, et al: Diffusion tensor imaging of the hippocampal formation in temporal lobe epilepsy. AJNR Am J Neuroradiol 24:1857-1862, 2003

3. Assaf Y, Basser PJ: Composite hindered and restricted model of diffusion (CHARMED) MR imaging of the human brain. Neuroimage 27:48-58, 2005

4. Basser PJ, Pajevic S, Pierpaoli C, Duda J, Aldroubi A: In vivo fiber tractography using DT-MRI data. Magn Reson Med 44:625-632, 2000

5. Beare R, Yang JY, Maixner WJ, Harvey AS, Kean MJ, Anderson VA, et al: Automated alignment of perioperative MRI scans: A technical note and application in pediatric epilepsy surgery. Hum Brain Mapp 37:3530-3543, 2016

6. Behrens TE, Berg HJ, Jbabdi S, Rushworth MF, Woolrich MW: Probabilistic diffusion tractography with multiple fibre orientations: what can we gain? Neuroimage 34:144-155, 2007

7. Behrens TE, Woolrich MW, Jenkinson M, Johansen-Berg H, Nunes RG, Clare S, et al: Characterization and propagation of uncertainty in diffusion-weighted MR imaging. Magn Reson Med 50:1077-1088, 2003

8. Berman JI, Berger MS, Chung SW, Nagarajan SS, Henry RG: Accuracy of diffusion tensor magnetic resonance imaging tractography assessed using intraoperative subcortical stimulation mapping and magnetic source imaging. J Neurosurg 107:488-494, 2007

9. Farina L, Bergqvist C, Zimmerman RA, Haselgrove J, Hunter JV, Bilaniuk LT: Acute diffusion abnormalities in the hippocampus of children with new-onset seizures: the development of mesial temporal sclerosis. Neuroradiology 46:251-257, 2004

10. Farquharson S, Tournier JD, Calamante F, Fabinyi G, Schneider-Kolsky M, Jackson GD, et al: White matter fiber tractography: why we need to move beyond DTI. J Neurosurg 118:1367-1377, 2013

11. Feinberg DA, Reese TG, Wedeen VJ: Simultaneous echo refocusing in EPI. Magn Reson Med 48:1-5, 2002

12. Fernandez-Miranda JC: Editorial. Beyond diffusion tensor imaging. J Neurosurg 118:1363-1366, 2013

13. Fritz CO, Morris PE, Richler JJ: Effect size estimates: current use, calculations, and interpretation. J Exp Psychol Gen 141:2-18, 2012

14. Golby AJ, Kindlmann G, Norton I, Yarmarkovich A, Pieper $\mathrm{S}$, Kikinis R: Interactive diffusion tensor tractography visualization for neurosurgical planning. Neurosurgery 68:496505,2011

15. Govindan RM, Makki MI, Sundaram SK, Juhász C, Chugani HT: Diffusion tensor analysis of temporal and extra-temporal lobe tracts in temporal lobe epilepsy. Epilepsy Res 80:3041,2008 
16. Gross DW, Bastos A, Beaulieu C: Diffusion tensor imaging abnormalities in focal cortical dysplasia. Can J Neurol Sci 32:477-482, 2005

17. Hagler DJ Jr, Ahmadi ME, Kuperman J, Holland D, McDonald CR, Halgren E, et al: Automated white-matter tractography using a probabilistic diffusion tensor atlas: application to temporal lobe epilepsy. Hum Brain Mapp 30:1535-1547, 2009

18. Hall WA, Truwit CL: Intraoperative MR-guided neurosurgery. J Magn Reson Imaging 27:368-375, 2008

19. Hill DL, Maurer CR Jr, Maciunas RJ, Barwise JA, Fitzpatrick JM, Wang MY: Measurement of intraoperative brain surface deformation under a craniotomy. Neurosurgery 43:514-528, 1998

20. Jansen FE, Braun KP, van Nieuwenhuizen O, Huiskamp G, Vincken KL, van Huffelen AC, et al: Diffusion-weighted magnetic resonance imaging and identification of the epileptogenic tuber in patients with tuberous sclerosis. Arch Neurol 60: $1580-1584,2003$

21. Jansons KM, Alexander DC: Persistent angular structure: new insights from diffusion MRI data. Dummy version. Inf Process Med Imaging 18:672-683, 2003

22. Jeurissen B, Leemans A, Jones DK, Tournier JD, Sijbers J: Probabilistic fiber tracking using the residual bootstrap with constrained spherical deconvolution. Hum Brain Mapp 32:461-479, 2011

23. Jeurissen B, Leemans A, Tournier JD, Jones DK, Sijbers J: Estimating the number of fiber orientations in diffusion MRI voxels: a constrained spherical deconvolution study. Proc Intl Soc Mag Reson Med 18:573, 2010 (Abstract)

24. Ji S, Roberts DW, Hartov A, Paulsen KD: Brain-skull contact boundary conditions in an inverse computational deformation model. Med Image Anal 13:659-672, 2009

25. Jones DK: Studying connections in the living human brain with diffusion MRI. Cortex 44:936-952, 2008

26. Kamada K, Todo T, Masutani Y, Aoki S, Ino K, Takano T, et al: Combined use of tractography-integrated functional neuronavigation and direct fiber stimulation. J Neurosurg 102:664-672, 2005

27. Kimiwada T, Juhász C, Makki M, Muzik O, Chugani DC, Asano E, et al: Hippocampal and thalamic diffusion abnormalities in children with temporal lobe epilepsy. Epilepsia 47:167-175, 2006

28. Lawes IN, Barrick TR, Murugam V, Spierings N, Evans DR, Song M, et al: Atlas-based segmentation of white matter tracts of the human brain using diffusion tensor tractography and comparison with classical dissection. Neuroimage 39:62-79, 2008

29. Le Bihan D, Poupon C, Amadon A, Lethimonnier F: Artifacts and pitfalls in diffusion MRI. J Magn Reson Imaging 24:478-488, 2006

30. Lipson AC, Gargollo PC, Black PM: Intraoperative magnetic resonance imaging: considerations for the operating room of the future. J Clin Neurosci 8:305-310, 2001

31. Maesawa S, Fujii M, Nakahara N, Watanabe T, Wakabayashi T, Yoshida J: Intraoperative tractography and motor evoked potential (MEP) monitoring in surgery for gliomas around the corticospinal tract. World Neurosurg 74:153161,2010

32. Mang SC, Gembris D, Grodd W, Klose U: Comparison of gradient encoding directions for higher order tensor diffusion data. Magn Reson Med 61:335-343, 2009

33. Maurer CR Jr, Hill DL, Martin AJ, Liu H, McCue M, Rueckert $\mathrm{D}$, et al: Investigation of intraoperative brain deformation using a 1.5-T interventional MR system: preliminary results. IEEE Trans Med Imaging 17:817-825, 1998

34. Mikuni N, Okada T, Enatsu R, Miki Y, Hanakawa T, Urayama S, et al: Clinical impact of integrated functional neuronavigation and subcortical electrical stimulation to preserve motor function during resection of brain tumors. J Neurosurg 106:593-598, 2007

35. Moeller S, Yacoub E, Olman CA, Auerbach E, Strupp J, Harel N, et al: Multiband multislice GE-EPI at 7 tesla, with 16-fold acceleration using partial parallel imaging with application to high spatial and temporal whole-brain fMRI. Magn Reson Med 63:1144-1153, 2010

36. Mori S, Crain BJ, Chacko VP, van Zijl PC: Three-dimensional tracking of axonal projections in the brain by magnetic resonance imaging. Ann Neurol 45:265-269, 1999

37. Nabavi A, Black PM, Gering DT, Westin CF, Mehta V, Pergolizzi RS Jr, et al: Serial intraoperative magnetic resonance imaging of brain shift. Neurosurgery 48:787-798, 2001

38. Nimsky C: Fiber tracking-we should move beyond diffusion tensor imaging. World Neurosurg 82:35-36, 2014

39. Nimsky C, Ganslandt O, Cerny S, Hastreiter P, Greiner G, Fahlbusch R: Quantification of, visualization of, and compensation for brain shift using intraoperative magnetic resonance imaging. Neurosurgery 47:1070-1080, 2000

40. Nimsky C, Ganslandt O, Fahlbusch R: Implementation of fiber tract navigation. Neurosurgery 61 (1 Suppl):306-318, 2007

41. Nimsky C, Ganslandt O, Hastreiter P, Wang R, Benner T, Sorensen AG, et al: Intraoperative diffusion-tensor MR imaging: shifting of white matter tracts during neurosurgical procedures-initial experience. Radiology 234:218-225, 2005

42. Nimsky C, Ganslandt O, Hastreiter P, Wang R, Benner T, Sorensen AG, et al: Preoperative and intraoperative diffusion tensor imaging-based fiber tracking in glioma surgery. Neurosurgery 61 (1 Suppl): 178-186, 2007

43. Nimsky C, Ganslandt O, Merhof D, Sorensen AG, Fahlbusch $\mathrm{R}$ : Intraoperative visualization of the pyramidal tract by diffusion-tensor-imaging-based fiber tracking. Neuroimage 30:1219-1229, 2006

44. Parker GJ, Haroon HA, Wheeler-Kingshott CA: A framework for a streamline-based probabilistic index of connectivity (PICo) using a structural interpretation of MRI diffusion measurements. J Magn Reson Imaging 18:242-254, 2003

45. Paulsen KD, Miga MI, Kennedy FE, Hoopes PJ, Hartov A, Roberts DW: A computational model for tracking subsurface tissue deformation during stereotactic neurosurgery. IEEE Trans Biomed Eng 46:213-225, 1999

46. Reese TG, Benner T, Wang R, Feinberg DA, Wedeen VJ: Halving imaging time of whole brain diffusion spectrum imaging and diffusion tractography using simultaneous image refocusing in EPI. J Magn Reson Imaging 29:517-522, 2009

47. Reinges MH, Nguyen $\mathrm{HH}$, Krings T, Hütter BO, Rohde V, Gilsbach JM: Course of brain shift during microsurgical resection of supratentorial cerebral lesions: limits of conventional neuronavigation. Acta Neurochir (Wien) 146:369377, 2004

48. Roberts DW, Miga MI, Hartov A, Eisner S, Lemery JM, Kennedy FE, et al: Intraoperatively updated neuroimaging using brain modeling and sparse data. Neurosurgery 45:1199_ 1207, 1999

49. Romano A, D’Andrea G, Calabria LF, Coppola V, Espagnet CR, Pierallini A, et al: Pre- and intraoperative tractographic evaluation of corticospinal tract shift. Neurosurgery 69:696-705, 2011

50. Salmenpera TM, Simister RJ, Bartlett P, Symms MR, Boulby PA, Free SL, et al: High-resolution diffusion tensor imaging of the hippocampus in temporal lobe epilepsy. Epilepsy Res 71:102-106, 2006

51. Skrinjar O, Nabavi A, Duncan J: Model-driven brain shift compensation. Med Image Anal 6:361-373, 2002

52. Tournier JD, Calamante F, Connelly A: MRtrix: diffusion tractography in crossing fiber regions. Int J Imaging Syst Technol 22:53-66, 2012

53. Tournier JD, Calamante F, Connelly A: Robust determination 
of the fibre orientation distribution in diffusion MRI: nonnegativity constrained super-resolved spherical deconvolution. Neuroimage 35:1459-1472, 2007

54. Tournier JD, Calamante F, Gadian DG, Connelly A: Direct estimation of the fiber orientation density function from diffusion-weighted MRI data using spherical deconvolution. Neuroimage 23:1176-1185, 2004

55. Tournier JD, Mori S, Leemans A: Diffusion tensor imaging and beyond. Magn Reson Med 65:1532-1556, 2011

56. Tuch DS: Q-ball imaging. Magn Reson Med 52:1358-1372, 2004

57. Wedeen VJ, Hagmann P, Tseng WY, Reese TG, Weisskoff RM: Mapping complex tissue architecture with diffusion spectrum magnetic resonance imaging. Magn Reson Med $\mathbf{5 4 : 1 3 7 7 - 1 3 8 6 , 2 0 0 5}$

58. Wieshmann UC, Symms MR, Shorvon SD: Diffusion changes in status epilepticus. Lancet 350:493-494, 1997

59. Zhong J, Petroff OA, Pleban LA, Gore JC, Prichard JW: Reversible, reproducible reduction of brain water apparent diffusion coefficient by cortical electroshocks. Magn Reson Med 37:1-6, 1997

\section{Disclosures}

Dr. Maixner receives royalties from Springer-Verlag for the books Pediatric Hydrocephalus and Spina Bifida.

\section{Author Contributions}

Conception and design: Yang, Beare. Acquisition of data: Yang, Maixner. Analysis and interpretation of data: all authors. Drafting the article: Yang, Beare. Critically revising the article: all authors. Reviewed submitted version of manuscript: all authors. Approved the final version of the manuscript on behalf of all authors: Yang. Statistical analysis: Yang, Beare. Administrative/technical/material support: Seal, Anderson, Maixner. Study supervision: Seal, Anderson, Maixner.

\section{Supplemental Information Videos}

Video 1. https://vimeo.com/195798997.

\section{Online-Only Content}

Supplemental material is available with the online version of the article.

Supplementary Document and Tables. https://thejns.org/doi/ suppl/10.3171/2016.11.PEDS16312.

\section{Previous Presentations}

Portions of this work were presented in poster form at the Organization for Human Brain Mapping (OBHM) annual meeting, Seattle, Washington, June 16-20, 2013, and as components of an invited lecture at the 15th World Federation of Neurosurgical Societies (WFNS) World Congress of Neurosurgery, Seoul, South Korea, September 8-13, 2013.

\section{Correspondence}

Joseph Yuan-Mou Yang, Department of Neurosurgery, Royal Children's Hospital, Neuroscience Research, Murdoch Childrens Research Institute, 50 Flemington Rd., Parkville, Victoria 3052, Australia.email: joseph.yang@mcri.edu.au. 